\title{
Trayectoria de las prácticas investigativas en la División de Educación Básica de la Universidad Nacional: Ruptura, innovación y cambio de paradigmas
}

\author{
Tracking Research Practices within the Elementary Education Division (DEB) at Universidad Nacional: \\ Rupture, Innovation, and Change of Paradigms
}

\author{
Cecilia Dobles-Trejos ${ }^{1}$ \\ Universidad Nacional \\ Centro de Investigación y Docencia en Educación \\ Heredia, Costa Rica \\ ceciliadobles347@gmail.com \\ Rafael Esteban Jiménez-Corrales ${ }^{2}$ \\ Universidad Nacional \\ Centro de Investigación y Docencia en Educación \\ Heredia, Costa Rica \\ rejc22@gmail.com \\ Lillian Susana Ruiz-Guevara \\ Universidad Nacional \\ Centro de Investigación y Docencia en Educación \\ Heredia, Costa Rica \\ susanaruiz@gmail.com \\ Marie Claire Vargas-Dengo 4 \\ Universidad Nacional \\ Centro de Investigación y Docencia en Educación \\ Heredia, Costa Rica \\ marie_d_claire@yahoo.com
}

\begin{abstract}
${ }^{1}$ Antropóloga social. Actualmente es profesora e investigadora del Centro de Investigación y Docencia en Educación (CIDE) de la Universidad Nacional en Costa Rica, sus funciones como académica se concentran en las áreas de investigación en educación inclusiva y acción social. Además ha sido profesora de la Escuela de Antropología y Sociología de la Universidad de Costa Rica, espacio en lo que ha trabajado en las áreas de gestión de la investigación y acción social. Ha realizado investigación en poblaciones específicas en temáticas como trabajo infantil, derechos indígenas y participación ciudadana; así como trabajos investigación y gestión sobre patrimonio cultural inmaterial.

2 Doctor en Educación con mención en Mediación Pedagógica. Máster en Evaluación Educativa y Licenciado en Historia. Ha ocupado cargos de subdirector de la División de Educación Básica del Centro de Investigación y Docencia en Educación (CIDE) de la Universidad Nacional, Costa Rica y coordinador de la Maestría en Pedagogía con énfasis en Diversidad en los procesos Educativos. Además, formó parte del equipo de Diseño Curricular de la Dirección de Docencia en esa misma institución. Actualmente, se desempeña como docente, investigador en la División de Educación Básica del Centro de Investigación y Docencia en Educación (CIDE) de la Universidad Nacional, Costa Rica.

${ }^{3}$ Doctora en Educación de la Universidad Estatal a Distancia, Costa Rica. Máster en Administración Educativa de la Universidad de La Salle y Licenciada en Educación Preescolar de la Universidad Nacional, Costa Rica. Académica de la División de Educación Básica (DEB) del Centro de Investigación y Docencia en Educación (CIDE) de la Universidad Nacional (UNA) en Costa Rica. Investigadora de temáticas relacionadas con la transición de la primaria a la secundaria, acreditación de carreras de Educación, creencias en la práctica docente. Forma parte del proyecto de gestión académico administrativa "La autoevaluación como estrategia de fortalecimiento de la calidad", así como de la Comisión de Trabajos Finales de Graduación de la de la DEB-CIDE, UNA.

${ }^{4}$ Doctora en Educación del Programa Latinoamericano de Doctorado en Educación, Universidad de Costa Rica. Máster en Currículo e Instrucción con énfasis en Educación Especial Bilingüe de The George Washington University, USA. Académica investigadora de la División de Educación Básica (DEB) del Centro de Investigación y Docencia en Educación (CIDE) de la Universidad Nacional, Costa Rica. Actualmente forma parte de la Comisión de Trabajos Finales de Graduación de la DEB-CIDE, UNA.
\end{abstract}


doi: http://dx.doi.org/10.15359/ree.19-2.21

URL: http://www.una.ac.cr/educare

CORREO: educare@una.cr

Recibido 17 de noviembre de 2014 • Corregido 23 de marzo de 2015 • Aceptado 27 de abril de 2015

Resumen. Este artículo aborda los distintos paradigmas y tendencias en las prácticas investigativas que se han desarrollado en la División de Educación Básica (DEB) del Centro de Investigación y Docencia en Educación (CIDE) de la Universidad Nacional (UNA) en Costa Rica, las cuales han marcado distintas tendencias en la forma de pensar y hacer investigación en la División. Estas prácticas constituyen un proceso, generado y desarrollado a lo largo del tiempo, que ha marcado una constante curricular histórica en la dinámica académica de la División, lo cual se ve reflejado en los planes de estudio, los programas de cursos y en los trabajos finales de graduación. En la década de 1990, la investigación en la DEB era de corte descriptivo-exploratoria, se caracterizaba por el análisis de datos centrado en la estadística descriptiva y la linealidad, propia de la corriente positivista. De forma paralela, se desarrollaron acciones de práctica educativa vinculadas con la extensión social en comunidades de atención prioritaria; emerge una línea de investigación cualitativa. La tendencia investigativa vigente en la División, se enmarca en el paradigma de investigación cualitativa, el cual posibilita la circularidad y recursividad investigativa. Se concluye que esta tendencia investigativa abre nuevas posibilidades en relación con las demandas sociopedagógicas actuales así como con el desarrollo de distintas capacidades idóneas para la formación docente preocupada por responder a las problemáticas socio-educativas y a la transformación social.

Palabras claves. Educación básica, enseñanza superior, investigación, tesis, universidad.

Abstract. This paper established the different paradigms and trends in research practices that take place within the División de Educación Básica (Elementary Education Division-DEB, for its name in Spanish) of Centro de Investigacion y Docencia en Educación (CIDE, from its name in Spanish) at Universidad Nacional (UNA) in Costa Rica. As such, these research practices have established diverse trends towards conceiving and doing research within DEB. As a process, these practices have been created and developed throughout time while establishing a historical constant in the academic curricular dynamics of DEB -as it can be observed in the study career programs, course syllabus and final graduation projects as well. In the 1990s, research at DEB was mainly descriptive-exploratory and it was characterized by data analysis focused on descriptive statistics and linearity from a positivist approach. At the same time, educational practices linked to a social approach in communities at risk were developed, and a quality-oriented research line emerged. Currently, research work at DEB is developed from a qualitative paradigm approach, thus allowing research circularity and recursiveness. It is then concluded, that this research trend allows new possibilities regarding current socio-pedagogical demands as well as developing teaching abilities to respond to socio educational problems and social transformation.

Keywords. Basic education, higher education, research, theses, university. 
doi: http://dx.doi.org/10.15359/ree.19-2.21

URL: http://www.una.ac.cr/educare

CORREO: educare@una.cr

\section{Las prácticas investigativas en la División de Educación Básica: Una constante en el proceso curricular y un cambio de paradigma investigativo}

Dentro de la dinámica de la División de Educación Básica (DEB) del Centro de Investigación y Docencia en Educación (CIDE), las prácticas investigativas no son un proceso que haya nacido en los últimos años; por el contrario, conforman un proceso que se ha gestado y desarrollado a lo largo del tiempo, tal y como se ve reflejado en los planes de estudio, los programas de cursos y formulación de proyectos, así como en las distintas actividades socializadoras de sus propuestas, alcances y resultados. Como proceso, dichas prácticas investigativas se han constituido en una constante en la dinámica académica de la División.Y, como todo proceso curricular, la investigación ha vivido diferentes etapas, ha experimentado diferentes formas de entenderse y concretarse.

A partir de lo anterior, se puede afirmar que las prácticas investigativas han marcado distintas tendencias en la DEB, no solo en la forma de concebir y hacer investigación, sino también, en las formas de aproximación a la realidad, de acercamiento a los distintos espacios educativos y sociales. Con ello, se amplían las posibilidades de acción e incidencia social de la labor académica investigativa en aras de estrechar el lazo universidad-sociedad y cumplir con la función social que, según Dengo (2005), es concomitante a la universidad estatal latinoamericana.

El propósito de este apartado es delinear algunas de dichas tendencias desde una mirada curricular-histórica, con el fin de comprender, tal y como lo señala Posner (2005) -con la profundidad que posibilitan los métodos socio-históricos-, e intentar conocer cómo se desarrollan los procesos de propuestas curriculares y determinar la lógica de estos. Desde esta mirada, se hace un recorrido por la historia que han pautado los trabajos finales de graduación (TFG) en la DEB, concentrándonos en los hitos más importantes -sin el afán de crear una historiografía de la investigación en la DEB-. Así, el apartado aporta una contextualización de las distintas prácticas investigativas de la DEB, desde la cual las nuevas propuestas cobran el sentido de un proceso evolutivo y permiten una comprensión de la propuesta actual desde marcos mayores de explicación, sin omitir que lo anterior está fuertemente vinculado con la formación académica de estudiantes en las distintas carreras que se ofertan en la DEB.

\section{Vinculación de la actividad investigativa con las carreras y los planes de estudio: Un marco de referencia contextual}

Cabe recordar que, las carreras del CIDE para el año 1991 estaban estructuradas -grosso modopor un tronco común en ciencias de la educación. Este tronco común constituía una base para todos los énfasis, como era el caso de las carreras de Educación para I y II ciclos y Educación Preescolar -ambas de la DEB-, así como las de III ciclo y Educación Diversificada -impartidas en la División de Educología (DE)- y la de Orientación -en la División de Educación para el Trabajo (DET)--, entre otras. La investigación era uno de los elementos constitutivos dentro de este tronco común. 
doi: http://dx.doi.org/10.15359/ree.19-2.21

URL: http://www.una.ac.cr/educare

CORREO: educare@una.cr

En ese entonces, la actividad investigativa se caracterizaba por la linealidad; se trataba de una investigación hija de una tendencia positivista, ligada a la psicología experimental, de una forma de hacer investigación que trabaja con hipótesis estadísticas, grandes marcos teóricos, instrumentos directamente derivados de ellos y un análisis de datos centrado tanto en la estadística descriptiva, como también, en algunos casos, en la estadística inferencial paramétrica. No obstante, ese modelo de tronco común fue sufriendo cambios a lo interno del CIDE, con la particularidad de que poco a poco fue asumido por cada una de las carreras. De esta forma, la DEB fue atendiendo las necesidades que le planteaba dicho tronco con su propio profesorado. Lo mismo sucedió en cada una de las demás unidades académicas del Centro.

Para 1991, en los planes de las carreras de la DEB -sobre todo en los de I y II ciclos, y Educación Preescolar- se incluye un curso de investigación en el Plan de Estudio al finalizar el bachillerato, como también, dos cursos de investigación en el nivel de licenciatura. Es importante mencionar que, aun cuando estos cursos todavía formaban parte del tronco común del CIDE, eran ideados, diseñados e impartidos por académicas de la DEB. De hecho, la DEB es la primera unidad académica del CIDE que cuenta con un documento propio que pauta la elaboración de los TFG. Este primer Reglamento de Normas y Procedimientos para Trabajos Finales de Graduaciones elaborado por dos académicas de la DEB: León y Pereira (1991).

En este contexto, la DEB era la única división del Centro que para entonces contaba con un Reglamento de TFG y con su propio texto guía para su elaboración. Sin duda, esto generó una secuencia y una lógica en los procesos de formación docente, según se explica más adelante. Años después, en 1998, entra en vigencia un segundo Reglamento en la DEB, elaborado en este caso por tres académicas y un académico de la División, a saber, Gamboa, Miranda, Pereira y Soto (1998). Este documento se elabora con base en una revisión y adaptación del documento de León y Pereira (1991).

Los trabajos finales de graduación (TFG) de la DEB se basaban, entonces, en un modelo compuesto básicamente por los siguientes apartados: Tema; problema; justificación; antecedentes; objetivos (o preguntas exploratorias); marco teórico; marco metodológico, tipo de estudio (experimental, cuasi-experimental, explicativo o bien descriptivo-exploratorio); población y muestra; definición de términos; instrumentos; análisis o procesamiento de datos; conclusiones; recomendaciones y bibliografía. Y, el formato del trabajo se apegaba a los lineamientos de estilo establecidos por la American Psychological Association (APA).

En esa época la mayoría de los TFG se enrumban hacia la clasificación de tipo descriptivoexploratorio, lo cual se evidencia al analizar tesis de esa época. Lo exploratorio se sustenta en la escogencia de temas novedosos, puntual de aulas específicas; lo descriptivo, por su parte, en que la medición se ubica en niveles de medición nominales, o bien de razón. Sin embargo, aparecen muy pocos estudios que utilizan coeficientes de correlación, o bien, pruebas de hipótesis, 
análisis de varianza, regresión múltiple o análisis multifactoriales. Aunado a lo anterior, es notoria la ausencia de estudios que involucran una estadística no paramétrica, sobre todo aquellos que utilizan tablas binomiales. Al analizar la tendencia en la elaboración de estos trabajos, se observa que, de alguna forma, se busca bajar el nivel del análisis estadístico, con el fin de que el estudiantado -futuro personal docente- contaran con un instrumental no tan complejo, de tal manera que se pudieran desarrollar en el contexto aula y con problemas puntuales.

En relación con lo anterior, la figura 1 muestra la trayectoria e incidencia de los enfoques y tipos de investigación utilizados del año 1978 al 2013, según lo reporta la sistematización y el análisis de los marcos metodológicos de los TFG elaborados en el nivel de licenciatura de las distintas carreras de la División de Educación Básica del CIDE entre esos años.

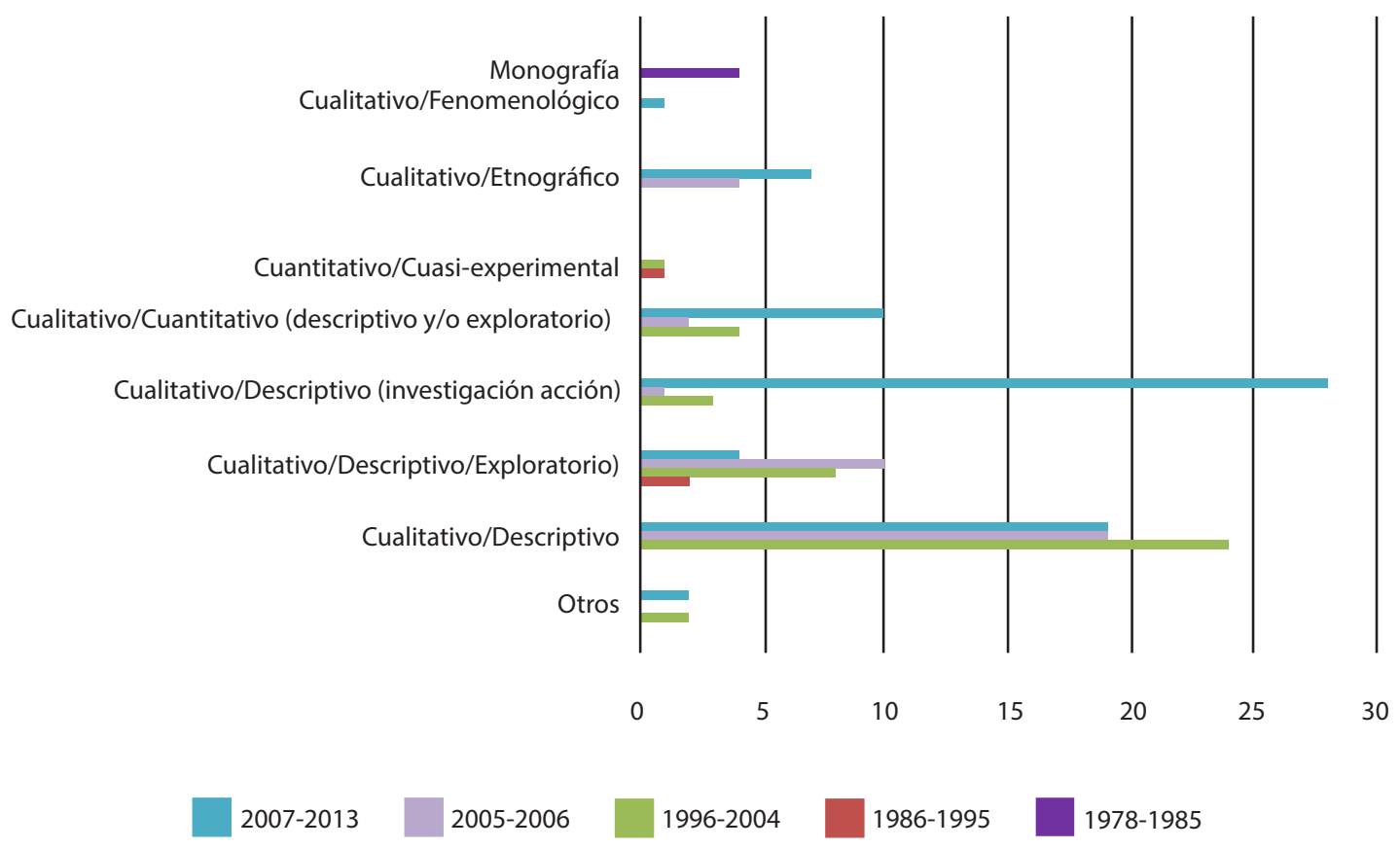

Figura 1. Enfoques y tipos de investigación utilizados en el marco metodológico del año 1978 al 2013 en la División de Educación Básica-CIDE-UNA. Elaboración propia.

El modelo investigativo del CIDE mantenía una definición conceptual, operacional e instrumental de variables. La DEB logra una primera ruptura con dicho modelo con el Reglamento elaborado por León y Pereira (1991), dado que este sustituye el planteamiento basado en hipótesis y su respectiva prueba, pasa a trabajar por objetivos y las variables son sustituidas por la definición de términos. 
doi: http://dx.doi.org/10.15359/ree.19-2.21

URL: http://www.una.ac.cr/educare

CORREO: educare@una.cr

En esta misma línea, Pacheco y Flores (1993) -también académicas de la DEB- aunaron experiencia y esfuerzos en la elaboración de una publicación de utilidad práctica para el estudiantado. Dicha publicación se editó por vez primera en la EUNA en el año 1993. Para el año 2003, el texto aún seguía reimprimiéndose y utilizándose tanto en universidades públicas y privadas, así como en instituciones de educación secundaria.

La importancia de esta publicación radica en que proporciona, al estudiantado, elementos básicos y funcionales para elaborar el trabajo investigativo; facilita la construcción de forma individual y promueve el autoaprendizaje. El texto ofrece una serie de conceptos, esquemasy actividades guiadas que facilitan desarrollar una investigación y recopilar información bibliográfica. Además, presenta una forma de realizar citas e incluye tanto el formato de ISBD como el APA, de una forma sumamente sencilla, simplificada y operativa. Esta publicación guarda una relación directa con los procesos de TFG de la DEB que se realizaban en esos años. La utilización del texto perseguía abrir espacios de reflexión para el estudiantado durante la elaboración de sus TFG. En este contexto, el documento es una evidencia de la concepción de investigación y de los TFG que se manejaba la División en ese momento.

Una revisión del documento en cuestión permite apreciar que el enfoque propuesto se define abiertamente como constructivista, sobre todo a partir de las preconcepciones e ideas previas de quienes hacen su lectura. En el texto se hace hincapié en que el modelo rompe con la tradición del paradigma positivista y enfatiza en la reflexión del estudiante en cada etapa. Específicamente, en el capítulo (unidad) IV se lee con toda claridad y nitidez el modelo de investigación que esbozan las autoras Pacheco y Flores (1993):

Cabe recalcar que el modelo que se presenta no corresponde al enfoque tradicional contenido en la mayoría de los libros que abordan la investigación desde un enfoque positivista.

Se le ofrece una alternativa para hacer investigación desde un paradigma constructivista. (p. 76)

El modelo propuesto, entonces, fue secuenciado de la siguiente forma: selección del tema; justificación; propósitos; preguntas exploratorias; marco teórico; marco metodológico; marco interpretativo y, por último, informes. En la publicación original, las etapas aparecen concatenadas por líneas que indican las relaciones; sin embargo, se mantiene cierta linealidad y los procesos reflexivos no aparecen explicitados, como se observa a continuación (ver figura 2 ( $^{5}$

${ }^{5}$ Las personas autoras de este artículo cuentan con la autorización de Pacheco y Flores (1993) para la adaptación de esta figura 2. 
doi: http://dx.doi.org/10.15359/ree.19-2.21

URL: http://www.una.ac.cr/educare

CORREO: educare@una.cr

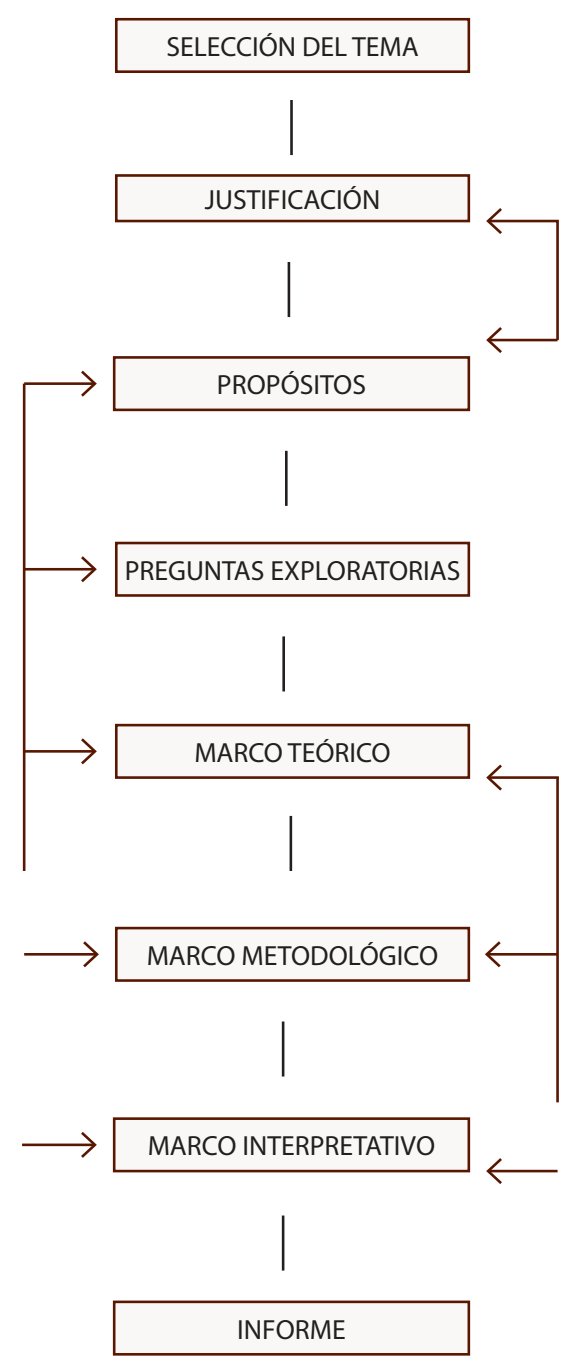

Figura 2. Etapas del proceso de investigación. Adaptación a partir de Pacheco y Flores (1993).

Para cada apartado se van aportando sugerencias clave que indican los aspectos a los cuales se debe responder en la elaboración del TFG. La lógica de este proceso investigativo se puede apreciar a continuación (ver tabla 1). 
doi: http://dx.doi.org/10.15359/ree.19-2.21

URL: http://www.una.ac.cr/educare

CORREO: educare@una.cr

Tabla 1

Elementos del modelo investigativo y sugerencias para su elaboración

\begin{tabular}{|c|c|}
\hline Elemento del modelo & Sugerencias dadas por las autoras para la elaboración de cada apartado \\
\hline Selección del tema & $\begin{array}{l}\text { 1. Aporte } \\
\text { 2. Interés personal } \\
\text { 3. Factibilidad }\end{array}$ \\
\hline Justificación & $\begin{array}{l}\text { 1. Interés institucional } \\
\text { 2. Interés personal } \\
\text { 3. Aporte } \\
\text { 4. Originalidad } \\
\text { 5. Responde al para qué de la investigación }\end{array}$ \\
\hline Propósitos & $\begin{array}{l}\text { 1. Punto de referencia que orientará la investigación. } \\
\text { 2. Intencionalidad } \\
\text { 3. Alcance } \\
\text { 4. Nivel de profundidad }\end{array}$ \\
\hline Preguntas exploratorias & $\begin{array}{l}\text { 1. Carácter exploratorio } \\
\text { 2. Ligadas al tema } \\
\text { 3. Relacionadas con los propósitos } \\
\text { 4. Flexibles } \\
\text { 5. Responde al qué de la investigación }\end{array}$ \\
\hline Marco teórico & $\begin{array}{l}\text { 1. Explícito con respecto al tema } \\
\text { 2. Susceptible de una revisión a lo largo del trabajo } \\
\text { 3. Incluye los antecedentes } \\
\text { 4. Se propone la siguiente secuencia de elaboración: Realidad - información } \\
\text { primaria o directa - información secundaria o indirecta } \\
4.1 \quad \text { Segundo nivel: Teorías e información empírica } \\
4.3 \quad \text { Tercer nivel: Fuentes informativas } \\
4.4 \quad \text { Valoración de la persona investigadora } \\
4.5 \text { Construcción del marco teórico. }\end{array}$ \\
\hline Marco metodológico & $\begin{array}{l}\text { 1. Responde al cómo de la investigación } \\
\text { 2. Sujetos } \\
\text { 2.1. Escogencia de la población } \\
\text { 2.1. Selección de la muestra } \\
\text { 2.3 Establecimiento del método de muestreo } \\
\text { 2.3. Determinación del tamaño de la muestra } \\
\text { 2.5. Representatividad, confiabilidad y validez } \\
\text { 3. Medios } \\
\text { 3.1 El Diseño de investigación } \\
\text { 3.2 Los instrumentos (válidos y confiables) } \\
\text { 3.3 Los procedimientos de análisis (revisión, ordenamiento y organización) }\end{array}$ \\
\hline
\end{tabular}




\begin{tabular}{ll}
\hline Elemento del modelo & Sugerencias dadas por las autoras para la elaboración de cada apartado \\
\hline Marco interpretativo & $\begin{array}{l}\text { 1. Establecer la consistencia entre marco teórico, marco metodológico y resultados } \\
\text { 2. Sintetizar y describir los resultados }\end{array}$ \\
& $\begin{array}{l}\text { 3.Analizar los resultados de acuerdo con los propósitos y preguntas exploratorias } \\
\text { 4. Realizar una síntesis general de los resultados }\end{array}$ \\
& 5. Relacionar la síntesis obtenida, con la realidad \\
6. Determinar las implicaciones del conocimiento generado
\end{tabular}

Nota: Elaboración propia de Jiménez a partir de Pacheco y Flores (1993).

Es oportuno reiterar que el texto de Pacheco y Flores (1993) se utilizó en los cursos de investigación de los niveles de bachillerato y de licenciatura y que, en su momento, se constituyó en la base de los TFG que se desarrollaban en la DEB. Sin embargo, si bien los cursos suscribían una propuesta de investigación acorde con dicho modelo que, para su momento proponía aspectos innovadores, la elaboración misma de los TFG seguía una lógica estructurada y el estudiantado debía amoldarse a las pautas dictadas por la Comisión de Trabajos Finales de Graduación (CTFG). De alguna forma, esto deja entrever rasgos paradigmáticos positivistas propios del momento de transición-, sobre todo en lo que se refiere a la metodología, ya que aún se seguía una lógica positivista.

En este modelo la población involucrada continuaba siendo ajena a la investigación, es decir, quien investigaba permanecía al margen de la dinámica investigada y sus reflexiones eran ante todo personales. A la luz de los años, quizá parezca un modelo muy lineal, pero bien se puede apreciar que para sus años de vigencia, proponía aspectos innovadores y, sobre todo, representaba un cambio en relación con el común de las prácticas investigativas del CIDE.

Cabe recordar que en medio de estos cambios, propuestas y búsqueda de formación en investigación, priva siempre una lógica y necesidad: Que las docentes formadas y los docentes formados en la DEB se convirtieran en investigadoras e investigadores. Es preciso indicar que las tesis de esa época abordan situaciones de aula y escuela de una forma muy clara. Debe recordarse que en ese momento existían convenios con el Ministerio de Educación Pública que nombraban estudiantes desde el ingreso a la carrera y al diplomado. Posteriormente eliminados los convenios, aún las estudiantes y los estudiantes se ubicaban rápidamente a laborar para el Ministerio de Educación. Por eso los proyectos de investigación se pensaban para aulas y escuelas, para grupos escolarizados. 
doi: http://dx.doi.org/10.15359/ree.19-2.21

URL: http://www.una.ac.cr/educare

CORREO: educare@una.cr

\section{La mirada investigativa puesta en la extensión educativa-social: Estrechando el enlace con las comunidades de atención prioritaria}

En los años entre 1990 y 1995, la DEB se aboca a comprender y responder a un reto de carácter nacional: la pobreza y las llamadas escuelas de atención prioritaria. Para ello, primero analiza y estudia distintas comunidades; luego se concentra específicamente en la comunidad de Guararí, en la provincia de Heredia, con la puesta en marcha de un proyecto de extensión, liderado por la Dirección de la División en ese momento a cargo de la M.Sc. LorenaVargas. Este proyecto buscaba, ante todo, dinamizar las escuelas marginales -de atención prioritaria-, mejorar la calidad de vida de los estudiantes y generar un impacto comunal a partir de la acción docente. Según Dobles-Trejos, Flores-Davis y Sisfontes-Guilarte (2015), dicho proyecto "no solo tenía un objetivo académico, sino que buscaba además impactar positivamente en la realidad educativa de Guararí" (p. 363). La lógica del proyecto era crear rutas, caminos, y promover la experiencia en el campo con la finalidad de posicionar a la DEB en un espacio que crecía a ritmos vertiginosos.

La comunidad de Guararí fue un espacio laboratorio para el quehacer de la DEB. A raíz de incursionar en este, se lograron vínculos con los licenciados Eugenio Espinoza y Hugo Hernández, fundadores del Programa de Mejoramiento de la Calidad de Vida en las Comunidades Urbano Marginales (PROMECUM). Este Programa se originó con una fuerte influencia de la DEB del CIDE. Inclusive, el ministro de Educación de entonces -Ing. Eduardo Doryan- había acordado, a manera de precontrato con la DEB, que esta División formara a las docentes que laborarían en el PROMECUN. Aunque dicho convenio no se llegó a firmar, cabe rescatar que el intento de una investigación más dirigida hacia la acción, la extensión y la transformación social se gestaba en la DEB. Sirva de ejemplo, que las prácticas pedagógicas del nivel de diplomado se realizaban, en una gran mayoría, en la comunidad de Guararí, de manera que las docentes se pudieran capacitar en la DEB.

No se pueden omitir otras acciones generadas en esta misma coyuntura, como las que se realizaron en la comunidad Finca San Juan, enmarcadas dentro de un proyecto de animación de lectura y conversatorios con docentes, a cargo del escritor Carlos Rubio Torres, con la ayuda del académico Rafael Jiménez Corrales. Así como la elaboración de la tesis doctoral del académico Rafael Espinoza Pizarro, la cual, siguiendo la lógica del accionar de la DEB, realizó en la comunidad de La Carpio.

Sin embargo, desde otra perspectiva, durante los años entre 1991 y 2000, siempre privó por parte de las autoridades de la DEB la tendencia de seguir una línea de investigación en el aula, focalizada totalmente en los aportes de Stenhouse (1987). En este período, se pensaba y se discutía que tanto el enfoque positivista como el cualitativo eran externos a la acción docente. Y que la propuesta de investigación en el aula de la escuela británica llevaría a la reflexión y al cambio. La reflexión, por tanto, debía centrarse en el currículo, en la evaluación y en la metodología. Incluso se generó una alianza con la Universidad de East Anglia y con el Centro Británico CARE, así como cursos y convenios para pasantes. 
A pesar de los lineamientos pautados por la Dirección de la DEB, la investigación tomó otro camino. Por el contrario, poco a poco fue cobrando fuerza, tanto una investigación cuantitativa más depurada, como una investigación cualitativa de una fuerte tendencia, que describía espacios, culturas, lógicas estudiantiles, pero no lograba la profunda reflexión de la acción docente. Al asumir la Dirección de la DEB Irma Zúñiga León, se“impulsa el desarrollo de la investigación, de la lectoescritura y de las relaciones interpersonales" (Doble-Trejos et al., 2015, p. 365), así como los procesos de autoevaluación, mejoramiento y acreditación de las distintas carreras de la DEB.

En años posteriores -2002-2004-, estando a cargo de la Dirección de la DEB la M. Sc. Herrera y de la Sub Dirección el Dr. Jiménez Corrales, se desarrollaron varias acciones de formación docente en comunidades de atención prioritaria, incluyendo la construcción de aulas en la escuela de la Gran Samaria (con financiamiento de la Fundación norteamericana EDUCAR), con la coordinación del M.Ed. Erick Quesada y el subdirector Dr. Rafael Jiménez Corrales. Estas acciones sirvieron de base al máster Quesada para la elaboración de un seminario de graduación, con un enfoque enmarcado desde la investigación-acción en un espacio de trabajo de una escuela y una comunidad de atención prioritaria en la región de Ciudad Quesada, en la provincia de Alajuela.

\section{La lógica curricular de los modelos antecedentes}

Cabe señalar que, en el periodo analizado, el mercado de empleo para docentes era muy amplio: el Ministerio de Educación Pública absorbía con facilidad a las graduadas y a los graduados; quienes se contrataban con diplomado, lograban plazas en propiedad con el nivel de bachillerato. Pocas universidades privadas habían considerado que formar en educación fuera un apropiado nicho de mercado. De esa forma, la UCR, la UNA y la UNED gozaban de un oligopolio en la formación de docentes.

La DEB generaba procesos de selección rigurosos para admitir estudiantes y los planes de estudios establecían que para iniciar el proceso de tesis o seminario de graduación había que terminar la totalidad de los cursos. Los anteproyectos eran rigurosamente revisados y se pedía un altísimo grado de elaboración. De ahí se explica la lógica de tener un curso de investigación cuantitativa y otro de investigación cualitativa. El curso de investigación de bachillerato reportaba niveles de repitencia de hasta un $60 \%$.

Desde la perspectiva curricular, tradicionalmente los procesos de formación en investigación generaban capacidades en los estudiantes, sobre todo las siguientes:

- Vinculación de la teoría con la práctica (en esa lógica)

- Profundización en las problemáticas de aula

- Elaboración detallada de instrumentos

- Manejo elemental de la estadística descriptiva 
doi: http://dx.doi.org/10.15359/ree.19-2.21

URL: http://www.una.ac.cr/educare

CORREO: educare@una.cr

No obstante, los modelos que se habían puesto en marcha a la fecha carecían de circularidad y recursividad; no se lograba desarrollar la capacidad autoreflexiva docente en los TFG. Después de concluir el proceso formativo y los TFG, quienes se graduaban -salvo en algunos casos- no seguían investigando; las tesis tardaban mucho tiempo en ser concluidas; había mucha repitencia en algunos cursos de investigación. En este marco, los procesos formativos e investigativos se tornaban sumamente mecánicos en su ejecución, la educación era vista como un proceso de aula, poco vinculada con situaciones sociohistóricas y comunales.

Con la apertura de la Maestría en Pedagogía con énfasis en Diversidad, en el año 2001, en la DEB se empezó a notar una carencia de nuevos abordajes metodológicos. La pedagogía, sobre todo española, base fundamental de la propuesta de posgrado, apuntaba a la necesidad de recurrir a la presencia de ampliar las capacidades del estudiantado para mirar los procesos de aula en forma más compleja. Sobre todo cuando se trataba de atender pedagógicamente fenómenos como la migración nicaragüense, la pobreza y otras dinámicas similares. Dicha Maestría tiene un enfoque pedagógico constructivista y sociocrítico (Belderbos, s. f.).

Cabe mencionar que, en la construcción del modelo de la maestría se contó con la participación del sociólogo, don Luis Fernando Riba -que de Dios goce- quien fue enfático en que el tema de cultura, sociedad y exclusión eran fundamentales para un cambio de dirección y enfoque. Su valioso aporte en este sentido fue sumamente significativo para la DEB. Por otra parte, los programas internacionales analizados para la elaboración del Plan de Estudio de la maestría apuntaban en esa misma línea. Los procesos que la maestría proponía trabajar no podían mirarse únicamente desde el aula, requerían una mirada más compleja y vinculante con los procesos sociales.

De esa reflexión nace la necesidad de contar con, por lo menos, una persona académics de formación de base en antropología. Piénsese que entre 1991 y el año 2000 la DEB había contratado ante todo docentes o funcionarios con títulos en ciencias de la educación, solo se contaba con un funcionario-en propiedad y de planta-formado enteramente en ciencias sociales. De esa forma, en las gestiones de Dirección-Subdirección Zúñiga-Herrera y Herrera-Jiménez, se empezó a intentar vincular profesionales de planta con otro perfil que complementaran la siempre fundamental visión pedagógica.

A esa situación se une la reflexión de estudiantes de licenciatura ante la ejecución de sus propios procesos de investigación y la problemática constante de reproducción curricular en el ejercicio docente. Y cada vez se empieza a mirar la formación de las carreras de grado ante la de posgrado, se notan diferencias significativas, no solo por el grado sino por la interdisciplinariedad de la formación. Además la maestría empieza a generar productos de investigación en tiempos menores que la licenciatura, de tal manera que, prácticamente terminando sus cursos, los estudiantes culminaban sus trabajos de graduación. Todos estos elementos conducen a plantear una nueva forma de mirar los procesos pedagógicos y a buscar nuevos senderos para las carreras de grado. 
A partir del interés de que el estudiantado pueda fortalecer los conocimientos en investigación construidos durante su formación en las distintas carreras de la DEB, en el 2008 se toma la decisión de realizar cambios en la forma en que estudiantes y docentes conciben y plantean sus propuestas de TFG. Se plantea, entonces, un nuevo proceso a seguir para hacer investigación en los niveles de licenciatura de la DEB. A continuación (ver tabla 2) se muestra de forma comparativa la propuesta investigativa anterior al año 2008 en relación con la propuesta investigativa de la posterior al año 2008 de la DEB:

Tabla 2

Propuestas investigativas de la DEB: Propuesta anterior al año 2008 y propuesta posterior al año 2008

Propuesta investigativa de la DEB anterior al año 2008 Propuesta investigativa de la DEB posterior al año 2008

La relación investigación-proceso formativo

Existe un salto entre la conclusión de los cursos con un modelo de trabajo en el aula universitaria a asumirse como investigadores sin las destrezas y apoyo suficiente.

Los cursos de investigación y el proceso de investigación se conciben de forma separada, independiente entre sí.

La investigación no está incorporada a la identidad del pedagogo.

Los cursos de Investigación I y II no se aprovechan suficientemente para la elaboración de los TFG.
Se parte del principio de que la investigación se debe enseñar y aprender en la práctica.

Los cursos de investigación y el proceso de investigación se dan de forma paralela:

a. Por tanto es un proceso pedagógico en el que se forman estudiantes en destrezas profesionales, incluyendo la investigación como algo orgánico y no como un añadido.

b. Se revisa el fortalecimiento de la profundización en las posturas teóricas y epistemológicas de estudiantes de licenciatura desde su rama profesional.

La investigación se asume como parte del proceso de formación, donde el estudiantado tiene posibilidades para asumirse a sí mismo o a sí misma como investigador o investigadora mediante:

a. Una auto aceptación de sus destrezas profesionales.

b. El reconocimiento de que puede aportar a su profesión.

Se aprovecha el conocimiento adquirido en los cursos de Investigación I y II en función de la elaboración de los trabajos finales de graduación en cuanto a que:

a. Sean más teórico-prácticos.

b. Creen destrezas investigativas. 
doi: http://dx.doi.org/10.15359/ree.19-2.21

URL: http://www.una.ac.cr/educare

CORREO: educare@una.cr

Propuesta investigativa de la DEB anterior al año 2008 Propuesta investigativa de la DEB posterior al año 2008

De la forma de concebir los anteproyectos de los TFG

El anteproyecto del TFG se concibe únicamente como un producto final resultado de un curso.

La presentación del anteproyecto se ubica al finalizar el I ciclo lectivo y el curso de Investigación I en el nivel de licenciatura.

a. Si el anteproyecto es aprobado, se deben incluir correcciones.

b. Los estudiantes y las estudiantes dedican una parte importante del tiempo a incluir correcciones, dando continuidad a su proceso de investigación.
El anteeproyecto se visualiza (estudiante y tutor) como parte inicial de un proceso de investigación.

El anteproyecto se presenta hasta finalizar el plan estudios de la licenciatura.

a. Si el anteproyecto se rechaza, no se cuenta con mecanismos de seguimiento a esta situación, pues ya no hay un curso que lo respalde.

b. Se atrasa el proceso investigativo.

De la forma de concebir el TFG

El TFG es visto como producto final de investigación.

Los conocimientos de investigación, aprendidos en años anteriores durante la carrera, no pueden ser vinculados con la práctica de hacer investigación al momento de plantear sus TFG.

La estructura para el diseño de trabajos finales de graduación es muy rígida.

Los TFG que ya están concluidos, presentan muchas inconsistencias teórico-metodológicas, entre ellas:

a. No hay una base epistemológica: consciencia de los investigadores e investigadoras de cuál es su postura ante la realidad investigada.

b. Combinación de paradigmas investigativos sin una sustentación clara.

c. Análisis de resultados no refleja dominio de teoría.

d. No hay un posicionamiento crítico en la elaboración del marco teórico.
Se rompe la noción de producto de investigación y se replantea la visión de que el TFG es un proceso formativo.

Se posibilita la vinculación con la práctica y los conocimientos de investigación aprendidos en años anteriores durante la carrera para plantear los TFG.

La estructura del TFG da espacio a reconocer nuevas posturas ante la investigación: más posmodernas.

A su conclusión, los TFG presentan mayor consistencia teórico-metodológicas, entre ellas:

a. Hay una base epistemológica: conciencia de los investigadores y las investigadoras de cuál es su postura ante la realidad investigada.

b. Se sustenta claramente la combinación de paradigmas investigativos.

c. El análisis de resultados refleja manejo teórico.

d. Se evidencia posicionamiento crítico en la elaboración del marco teórico. 


\section{Propuesta investigativa de la DEB anterior al año 2008 Propuesta investigativa de la DEB posterior al año 2008}

De los estudiantes y las estudiantes

No ha toman conciencia de sus habilidades como profesionales:

a. No saben que saben.

b. No tienen una postura clara ante la realidad educativa (debilidad en la reflexión crítica de la realidad).

No logran apropiarse de sus procesos de investigación, pues eran vistos como ejercicios mecánicos que debían seguir las directrices propuestas por el tutor o la tutora.

No se cuestionan o preguntan sobre su quehacer pedagógico y el impacto que tiene en la realidad educativa nacional.

No tienen la curiosidad de buscar y conocer nuevas realidades que les permitan explorar sus potencialidades como futuros y futuras profesionales.

La investigación es vista como un "tabú": es algo feo, incómodo, tedioso, mucho trabajo innecesario, no está vinculada al quehacer docente.

Hay mayor claridad en los grupos de estudiantes del papel tutorial en el TFG por lo que:

a. Se asume como acompañante en el proceso.

b. Por consecuencia, los TFG se realizan como un proceso de investigación.
Toman conciencia de sus habilidades docentes profesionales:

a. Se empoderan (saben que saben).

b. Son capaces de tomar postura y visión crítica ante la realidad socioeducativa.

c. Se apropian del objeto de investigación.

Los estudiantes y las estudiantes asumen su papel como investigadores o investigadoras, pues tienen mayor claridad en qué consiste una investigación.

Se cuestionan o preguntan sobre su quehacer pedagógico y el impacto que tiene en la realidad educativa nacional.

Se les despierta la curiosidad y capacidad de asombro ante nuevas realidades, desde donde exploran sus habilidades como futuros y futuras profesionales docentes.

Vivencian el proceso investigativo y las posibilidades que este les abre de involucramiento e impacto en la realidad social y educativas desde su quehacer docente.

No hay claridad en los estudiantes sobre el papel de tutoría del TFG por lo que:

a. Se asume como docente de curso.

b. Por consecuencia, los trabajos finales de graduación se realizan como una sumatoria de tareas. 
doi: http://dx.doi.org/10.15359/ree.19-2.21

URL: http://www.una.ac.cr/educare

CORREO: educare@una.cr

Propuesta investigativa de la DEB anterior al año 2008 Propuesta investigativa de la DEB posterior al año 2008

De los tutores y las tutoras de TFG

El personal tutor está desvinculado del anteproyecto (desinformado sobre él), lo cual genera descoordinación y falta de entendimiento de cuál es la fase del proceso de investigación en que se encuentra (madurez del investigador-estudiante).

Muchas tutorías son asumidas como una inducción al proceso de elaboración de tesis y no como un mecanismo para fortalecer las destrezas y apropiación del proceso de investigación por parte del estudiante:

a. Si el tutor o la tutora falla, la tesis se descontinúa.

b. Se continua con la lógica de trabajo: "aula universitaria".

El estudiante o la estudiante no tiene motivación para continuar con su trabajo de investigación.

Los tutores y las tutoras asumen los anteproyectos de forma individual e independiente, por lo que:

a. No siempre reconocen la propuesta metodológica definida en el anteproyecto aprobado.

Si hay modificación de las propuestas metodológicas del anteproyecto, se genera más dependencia en sus estudiantes y crisis en la apropiación de "su" investigación.

Muchas tutorías eran asumidas como una inducción al proceso de elaboración de tesis y no como un mecanismo para fortalecer las destrezas y apropiación del proceso de investigación por parte del estudiantado:

a. Si el tutor o la tutora falla, la tesis se descontinúa.

b. Se continua con la lógica de trabajo:"aula universitaria".

c. El estudiante no tiene motivación para continuar con su trabajo de investigación.
Se prevé un acompañamiento oportuno de los tutores y tutoras desde el Il ciclo del nivel de licenciatura una vez que el anteproyecto está aprobado por la CTFG.

El acompañamiento de tutoría al estudiantado se da cuando aún está cursando la licenciatura para posibilitar:

a. Crear más autonomía en el proceso de TFG (apropiación de su propuesta).

b. Más habilidades de investigación.

c. Contar con más apoyo docente, pues aún no hay madurez suficiente para asumir el proceso de manera independiente.

Se proporciona un acompañamiento a los tutores y las tutoras durante el proceso y desde los cursos de investigación, lo cual permite una comprensión de la propuesta metodológica definida en el anteproyecto.

a. Esta lógica de tutorías rompe radicalmente con la lógica de formación que se siguió durante los cinco años de carrera en que se ha sido muy "directivo".

b. Durante el año se guía al estudiantado a generar un proceso de maduración ante la investigación.

Se coordina con personal tutor para que reconozca que sus estudiantes están avanzando en su proceso de formación:

a. Desarrollan habilidades en la elaboración de su metodología de investigación.

b. Desarrollan habilidades en la elaboración de instrumentos de investigación.

c. Desarrollan habilidades en el análisis de la información.

d. Son capaces de construir una propuesta teórica: crítica y analítica en el que toman en cuenta su postura ante la realidad.

Nota: Elaboración propia. 
doi: http://dx.doi.org/10.15359/ree.19-2.21

URL: http://www.una.ac.cr/educare

CORREO: educare@una.cr

\section{La necesidad de ampliar la mirada de los procesos formativos en investigación: Un llamado hacia nuevos abordajes metodológicos}

A partir del año 2008, en el nivel de licenciatura de las tres carreras de la DEB: Pedagogía con énfasis en I y II ciclos; Pedagogía con énfasis en Educación Preescolar y Educación Especial con énfasis en Integración, se desarrolla un modelo de investigación -compartido, en gran medida, por el Programa de Maestría en Pedagogía- el cual parte de la lógica del triángulo de la investigación propuesto por Flórez y Tobón (2001) y de la lógica de la espiral recursiva sostenida por los principios de la investigación-acción. El triángulo de investigación se ilustra a continuación (ver figura 3).

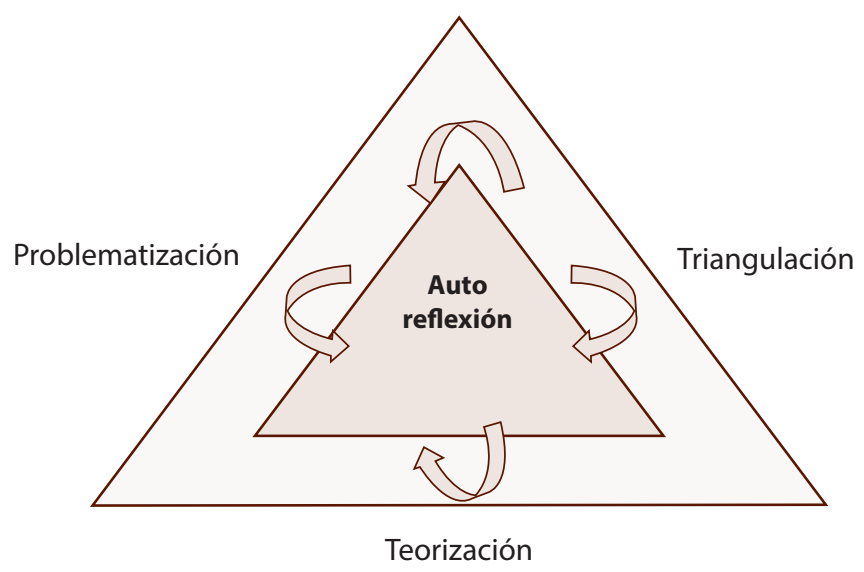

Figura 3. Triángulo de investigación. Elaboración propia.

Tobón establece que toda investigación nace de una situación -o reto- construida por el investigador o investigadora como parte de su necesidad de acople a la realidad,así como de su experiencia y reflexión, o bien, por una situación problema histórica. Esa situación reto, tiene dos caminos lógicos: uno, tomar hacia la teoría y el otro, ingresar al campo para su profundización, donde la autoreflexión tiene una ubicación central en el triángulo.

La actual Comisión de Trabajos Finales de Graduación (CTFG) de la DEB considera que el triángulo de la investigación es una forma de intentar mirar la complejidad, que se enmarca en el principio de incertidumbre. Las rutas de investigación no tienen una única lógica, como tampoco un origen único, sino que se trata de imaginar una figura recursiva -pues forma una grafía recursiva- sin principio ni fin, porque se encuentra dentro de la incertidumbre de la vida misma. Bien puede que se parta de un enfoque teórico, de una vivencia o inmersión en el campo, como también puede hacerse de un reto social, o bien, de dos vértices o de los tres. 
doi: http://dx.doi.org/10.15359/ree.19-2.21

URL: http://www.una.ac.cr/educare

CORREO: educare@una.cr

No obstante, en respuesta a las demandas sociopedagógicas que se debaten entre las oportunidades laborales de los futuros pedagogos y pedagogas, y la necesidad de dar una visión nueva a su quehacer docente, en la actualidad, en los cursos de investigación se ha intentado que el estudiantado se sumerja en distintos espacios pedagógicos o bien 'pedagogisables'. Esos espacios pueden ser las escuelas, las comunidades, o un determinado espacio educativo, institucional o no institucional, formal o no formal.

Inmerso en el campo, el estudiantado se sentirá en la necesidad de abordar el camino de 'atrapar esa realidad', para lo cual establecerá un diálogo, por un lado con sus conocimientos y formación universitaria y, por otro, con las realidades en las que tendrá como reto buscar caminos para mejorarla. El principio de espiral recursiva obliga a mirar el triángulo en múltiples dimensiones: cada recorrido o ruta hace que tanto teoría, situación-reto y realidad se modifiquen constantemente y modifiquen las capacidades cerebrales, las rutas y vías neuronales del aprendiente. Este principio recursivo nos remite a un recorrido en forma de espiral en ciclos el cual es concebido por K. Lewin (1890-1947) y luego desarrollado por diversos autores, entre ellos, Kemmis (1988) y Elliot (1993).

Desde esta perspectiva, el modelo en espiral genera distintas capacidades y, a la vez, da la posibilidad para que se desarrollen en el ser interno del estudiantado. Dentro de estas podemos mencionar las siguientes:

- Capacidad de aprender haciendo.

- Capacidad de ruptura de la linealidad con los modelos pedagógicos y de investigación anteriores.

- Capacidad de desarrollar una mirada desde la complejidad. Los niños, las niñas, o participantes se conciben en núcleos complejos, el participante es niño, comunidad, región, país, planeta y cosmos, pero a su vez es ser vivo, especie, se eleva a las estrellas y se engrandece en lo más microscópico de la vida.

- Capacidad de ruptura de las relaciones lineales teoría-práctica, para mirar relaciones complejas en una relación de práctica-teoría-práctica.

- Capacidad de vivir la incertidumbre. El modelo no parte de certezas; al ingresar al campo, el estudiantado va palpando la incertidumbre de la vida. Aprende formas de danzar con la incertidumbre; vive esta, en la vida académica y en su vida integral, y aprende a danzar con ella.

- Capacidad de vivenciar la vida en la voz y miradas de las otras personas. El ingreso al campo permite escuchar, mirar, darse cuenta de que la realidad es contingente, que se escribe desde miradas diversas, que más que juzgar, debe comprender e integrar. 
- Capacidad de ruptura de propuestas que transitan de la proclama a la demanda. De forma contraria a una investigación que parte de problemas preconcebidos por la teoría e implica una relación del poder del personal experto, en la ruta de la I-A el estudiantado busca comprender que para hacer efectivos los procesos pedagógicos, estos deben nacer de las demandas de los individuos participantes $y$, por tanto, es con ellos que debe generar propuestas de solución y procesos de concienciación.

- Capacidad reflexiva. Toda decisión del estudiantado en el hacer va generando una ruta metodológica alternativa, participativa, la cual incentiva la reflexión profunda con las otras personas y consigo mismo. Es decir, se aumenta su capacidad dialógica y auto-dialógica, lo que le va generando la conciencia de que es un ser autopoiético que comparte con seres igualmente autopoiéticos.

\section{Un nuevo cambio de rumbo en el quehacer docente-investigativo de la DEB: Rutas de investigación y saberes que se desarrollan desde el nuevo paradigma de investigación}

En el marco de la propuesta investigativa actual que se desarrolla en la DEB, el paradigma cualitativo de investigación expone a la División ante distintas posibilidades, tales como:

- Lectura del contexto socioeducativo y de los espacios educativos, sociales y culturales.

- Comprensión de los distintos espacios socioeducativos y culturales desde la coyuntura histórica.

- Comprensión del porqué se abordan las problemáticas educativas, sociales y humanas desde el paradigma cualitativo.

- Comprensión no fragmentada de la realidad: los espacios educativos, sociales y culturales en el marco de la globalidad contextual.

- Experiencia situada y vivencia directa en el campo: incursión en distintos espacios sociales y educativos formales y no formales.

- Dialogar con la teoría y articularla con la práctica: leer la realidad desde la teoría.

- Reconocer la mirada del 'otro'como participante en la investigación.

- Interacción sujeto-sujeto con las personas participantes.

- Intercambio de experiencias y su devolución con los actores y actoras en el proceso investigativo. 
doi: http://dx.doi.org/10.15359/ree.19-2.21

URL: http://www.una.ac.cr/educare

CORREO: educare@una.cr

Las posibilidades anteriormente desglosadas tienen una estrecha relación con los procesos que competen a una formación de docentes preocupada por responder a las problemáticas educativas y a la transformación social. En este sentido, cabe señalar que la elaboración de un TFG -desde la formulación del anteproyecto- requiere de distintos saberes y habilidades que se espera el estudiantado haya podido desarrollar durante el proceso formativo y a lo largo de la carrera que cursa en la DEB, dado que muchos de estos aspectos son inherentes a la formación profesional docente. Entre estos se mencionan los siguientes:

- Lectura de realidades socioeducativas y suproblematización de forma fundamentada y documentada.

- Incursión en espacios socioeducativos tradicionales y emergentes para su transformación desde procesos pedagógicos pertinentes.

- Identificación y planteamiento de un problema de investigación de pertinencia y relevancia social.

- Discusión en torno al tema y problema de investigación desde distintas posiciones, enfoques e investigaciones previas.

- Utilización y aplicación de estrategias de: observación, descripción, interacción con otras personas, entre otras.

- Apropiación al argumentar, sustentar e hilar las ideas tanto en la expresión oral como escrita.

- Definición de categorías conceptuales a partir de un tema específico: establecer vínculos entre ellas, rebatirlas, contrastarlas o integrarlas.

A partir de la sistematización y el análisis de un total de 29 anteproyectos presentados a la CTFG en el año 2013, por el estudiantado de las tres carreras de la DEB que cuentan con el nivel de licenciatura, a saber, Pedagogía con énfasis en I y II ciclos, Pedagogía con énfasis en Educación Preescolar y Educación Especial con énfasis en Integración, se concluye que muchos de los saberes y habilidades anteriormente señalados no siempre están desarrollados en el estudiante o la estudiante al llegar al nivel de licenciatura, por lo cual es necesario atender la importancia de fortalecerlos desde los distintos cursos que se ofrecen en las carreras de la División, desde los niveles de diplomado y bachillerato, con la finalidad de que el estudiante o la estudiante logre: 
- Trasladar conocimientos previos: lo aprendido en otros cursos y niveles de la carrera que cursa.

- Reconocer el proceso investigativo como un ciclo y proceso recursivo: visualizar una ruta a seguir compuesta por distintas fases, de forma similar a todo proceso pedagógico que tiene inicio, desarrollo, fin, reinicio, etc.

- Dimensionar los alcances del TFG que realiza en relación con la vivencia de sus participantes en términos de aportes y beneficios.

- Utilizar, de forma adecuada y actualizada, la terminología específica de la carrera y de la disciplina de estudio.

- Evidenciar coherentemente el posicionamiento paradigmático investigativo.

- Articular de forma coherente y concordante, los distintos componentes del anteproyecto y TFG.

Algunas habilidades más específicas involucradas en el proceso investigativo, las cuales también merecen atención en los procesos formativos, se refieren, por ejemplo, a la selección de técnicas de investigación adecuadas para recabar precisamente aquella información que se requiere; aprender a relacionarse con la información que se recaba para dialogar con ella tanto desde la teoría como desde la realidad misma -para no quedarse en una lectura de la realidad desde la inmediatez de la circunstancia cotidiana-; ejercitarse en el análisis de la información en relación con la identificación de patrones e inferencia de aspectos recurrentes en los datos, entre otras.

En relación con la búsqueda de información pertinente y el hallazgo de datos actualizados y relevantes al tema que se investiga, es necesario fortalecer la utilización de herramientas tecnológicas para la búsqueda en las bases de datos a las que la universidad tiene acceso, así como la aaplicación de estrategias de revisión bibliográfica.

Una valoración de los saberes presentes y ausentes en el estudiantado que realiza sus anteproyectos de TFG en la DEB señala que muchos son los retos que nos merece la formación de docentes, dado que muchos de los aspectos mencionados anteriormente son inherentes a la profesión docente y, por tanto, se esperaría que estos saberes y habilidades se desarrollen desde los distintos niveles formativos en una escuela de formación docente y durante estos, como lo es la División de Educación Básica del CIDE. 
doi: http://dx.doi.org/10.15359/ree.19-2.21

URL: http://www.una.ac.cr/educare

CORREO: educare@una.cr

\section{Referencias}

Belderbos, M. (s. f.). Maestría Pedagogía. Recuperado de http://www.cide-basica.una.ac.cr/ index.php?view=article\&catid=47\%3Amaestria-en-pedagogia\&id=53\%3Amaestriapedagogia\&format=pdf\&option=com content\&ltemid $=142$

Dengo, M. E. (2005). Desarrollo de las universidades latinoamericanas en el siglo XX y la acreditación. Cuadernos SINAES No 1. San José, Costa Rica: SINAES.

Dobles-Trejos, C., Flores-Davis, L. E. y Sisfontes-Guilarte, P. (2015). Diálogos y reconstrucción histórica de la División de Educación Básica. Revista Electrónica Educare, 19(1), 353-373. doi: http://dx.doi.org/10.15359/ree.19-1.19

Elliot, J. (1993). El cambio educativo desde la investigación acción. Madrid: Morata.

Gamboa, A., Miranda, G., Pereira, Z. y Soto, R. (1998). Reglamento de normas y procedimientos para la elaboración de trabajos finales de graduación de la División de Educación Básica del CIDE. Heredia: CIDE, Universidad Nacional. Manuscrito inédito.

Kemmis, S. (1989). El curriculum: Más allá de la teoría de la reproducción. Madrid: Morata.

León, A. y Pereira, Z. (1991). Reglamento de normas y procedimientos para trabajos finales de graduación de la División de Educación Básica. Heredia: CIDE, Universidad Nacional. Manuscrito inédito.

Posner, G. (2005). Análisis del currículo (3a ed.). Bogotá: McGraw-Hill.

Pacheco, A. T. y Flores, L. E. (1993). La investigación como proceso de construcción del conocimiento. Heredia: EUNA.

Flórez, R. y Tobón, A. (2001). Investigación educativa y pedagógica. Bogotá: McGraw-Hill.

Stenhouse, L. (1987). La investigación como base de la enseñanza. Madrid: Morata.

\section{Cómo citar este artículo en APA:}

Dobles-Trejos, C., Jiménez-Corrales, R. E., Ruiz-Guevara, L. S. y Vargas-Dengo, M. C. (Mayo-agosto, 2015). Trayectoria de las prácticas investigativas en la División de Educación Básica de la Universidad Nacional: Ruptura, innovación y cambio de paradigmas. Revista Electrónica Educare, 19(2), 383-404. doi: http:// dx.doi.org/10.15359/ree.19-2.21

Nota: Para citar este artículo en otros sistemas puede consultar el hipervínculo "Como citar el artículo" en la barra derecha de nuestro sitio web: http://www.revistas.una.ac.cr/index.php/EDUCARE/index 